\title{
SUM-ESSENTIAL GRAPHS OF MODULES
}

\author{
JERZY MATCZUK AND ALI MAJIDINYA
}

\begin{abstract}
The sum-essential graph $\mathcal{S}_{R}(M)$ of a left $R$-module $M$ is a graph whose vertices are all nontrivial submodules of $M$ and two distinct submodules are adjacent iff their sum is an essential submodule of $M$. Properties of the graph $\mathcal{S}_{R}(M)$ and its subgraph $\mathcal{P}_{R}(M)$ induced by vertices which are not essential as submodules of $M$ are investigated. The interplay between module properties of $M$ and properties of those graphs is studied.
\end{abstract}

\section{INTRODUCTION}

Throughout the paper a ring means a unital associative ring and a module is a left unitary module over a given ring $R$.

There are many studies on various graphs associated to modules, rings and other algebraic structures. The aim of the paper is to investigate the interplay between module properties of a module $M$ and properties of its sum-essential and proper sum-essential graphs. This will result in classification of modules in terms of some specific properties of those graphs.

For a given module $M$ the sum-essential graph of $M$ is a simple graph $\mathcal{S}_{R}(M)$ (i.e. unweighted, undirected graph containing no graph loops or multiple edges) whose vertices are nontrivial submodules (i.e. different from 0 and $M$ ) and two distinct vertices are adjacent if and only if the sum of the corresponding submodules is an essential submodule of $M$. We will also study the proper sum-essential graph of $M$ which is a subgraph of $\mathcal{S}_{R}(M)$ generated by vertices which, as submodules of $M$ are not essential.

The graph $\mathcal{S}_{R}(M)$ was introduced and studied by Amjadi in [5] in a very special case when $R$ is a commutative ring and $M={ }_{R} R$. There are other graphs relating submodule structure of a given module. For example comaximal left ideal graphs, i.e. graphs whose vertices are nontrivial left ideals of a ring $R$ and two vertices $I, J$ are adjacent if and only if $I+J=R$ were considered in [4, 9, 10, The intersection graph of a module, i.e. a simple graph whose vertices are nontrivial submodules and two distinct vertices are adjacent if the intersection of corresponding submodules is nonzero, was introduced and investigated in [8. Its complement graph was also examined. Earlier those graphs were considered in the context of left ideals of a given ring (see [2, 3]). In [1] the authors considered the inclusion graph whose vertices were nontrivial left ideals of a ring.

The first, introductional section contains elementary observations needed later on. We also show that $\mathcal{S}_{R}(M)$ and $\mathcal{P}_{R}(M)$ are connected graphs of diameter not bigger than 3.

Section 2 concentrates on modules $M$ such that the associated graphs contains vertices of small degree. Theorems 2.1 and 2.3 show that if every vertex of $\mathcal{S}_{R}(M)\left(\mathcal{P}_{R}(M)\right)$ is of finite degree then the graph has only finitely many vertices. An information about the structure of such modules is also given. The remaining part of this section is mainly devoted to investigation of modules $M$ such that $\mathcal{P}_{R}(M)$ contains a vertex of degree 1 . Theorem 2.13

2010 Mathematics Subject Classification. 05C25, 05C40, 16D99.

Key words and phrases. sum-essential graph, essential submodule, uniform dimension. 
offers necessary and sufficient condition for a submodule $U$ of $M$ to be of degree 1 as a vertex in $\mathcal{P}_{R}(M)$. It also appears (see Theorem 2.18) that either all submodules of degree one in $\mathcal{P}_{R}(M)$ are simple or $M$ contains the unique largest submodule of degree 1, examples are presented.

Section 3 concentrates on modules whose graphs are complete, $k$-regular and triangle-free or a tree. For example, Theorem 3.2 describes modules $M$ with complete graph $\mathcal{S}_{R}(M)$. Theorem 3.11 and 3.12 give necessary and sufficient conditions for $\mathcal{P}_{R}(M)$ to be triangle-free and a tree, respectively. The girth of $\mathcal{S}_{R}(M)$ and $\mathcal{P}_{R}(M)$ is also described.

\section{Preliminaries}

If not specified otherwise $M$ denotes a left module over a given ring $R$. We write $A \leqslant_{e} M$ if $A$ is an essential submodule of $M$ (i.e. if $A \cap B \neq 0$, for any nonzero submodule $B$ of $M), \operatorname{udim}(M)$ stands for the uniform dimension of $M$. For $m \in M$, ann $(m)$ denotes the annihilator of $m$ in $R$. The reader can consult [6] and [7] for precise definitions and properties needed in the text.

Let $G$ be a simple graph. The vertex set of $G$ is denoted by $\mathcal{V}(G), \operatorname{deg}_{G}(v)$ stands for the degree of $v \in \mathcal{V}(G)$, i.e. the cardinality of the set of all vertices which are adjacent to $v$. The maximum and minimum degrees of the graph $G$ are the maximum and minimum degree of its vertices and are denoted by $\Delta(G)$ and $\delta(G)$, respectively. $G$ is a complete graph if every pair of distinct vertices of $G$ are adjacent, $\mathcal{K}_{n}$ will stand for a complete graph with $n \in \mathbb{N}$ vertices. The graph $G$ is $k$-regular, if $\operatorname{deg}_{G}(v)=k<\infty$ for every $v \in \mathcal{V}(G)$.

Let $u, v \in \mathcal{V}(G)$. We say that $u$ is a universal vertex of $G$ if $u$ is adjacent to all other vertices of $G$ and write $u \backsim v$ if $u$ and $v$ are adjacent. The distance $d(u, v)$ is the length of the shortest path from $u$ to $v$ if such path exists, otherwise $d(x, y)=\infty$. The diameter of $G$ is $\operatorname{diam}(G)=\sup \{d(u, v) \mid u, v \in \mathcal{V}(G)\}$.

The girth of a graph $G$, denoted by $g(G)$, is the length of a shortest cycle in $G$. If $G$ has no cycles, then $g(G)=\infty$.

A subset $S \subseteq \mathcal{V}(G)$ is independent if no two vertices of $S$ are adjacent. For a positive integer $k$, a $k$-partite graph is a graph whose vertices can be partitioned into $k$ nonempty independent sets.

Let us recall that if $M$ is a left module over the ring $R$, then:

- The sum-essential graph of $M$ is a simple graph $\mathcal{S}_{R}(M)$ such that $\mathcal{V}\left(\mathcal{S}_{R}(M)\right)$ consists of all nontrivial submodules of $M$ and two vertices are adjacent if and only if the sum of the corresponding submodules is an essential submodule of $M$.

- The proper sum-essential graph of $M$, denoted by $\mathcal{P}_{R}(M)$, is a subgraph of $\mathcal{S}_{R}(M)$ induced by vertices which are not essential submodules of $M$.

Let us remark that $\mathcal{S}_{R}(M)$ has exactly one vertex iff $M$ contains a submodule $B$ such that both $B$ and $M / B$ are simple modules. On the other hand, if the graph $\mathcal{P}_{R}(M)$ is not empty, then the module $M$ is not uniform and hence $\left|\mathcal{V}\left(\mathcal{P}_{R}(M)\right)\right| \geq 2$.

Henceforth we will assume that all considered modules $M$ have at least two proper submodules, i.e. $\left|\mathcal{V}\left(\mathcal{S}_{R}(M)\right)\right| \geq 2$. Notice that if $N$ is a nontrivial nonessential submodule of $M$ then $N$, as a vertex of $\mathcal{S}_{R}(M)$ is adjacent to its complement and every vertex which is essential as a submodule of $M$ is a universal vertex of $\mathcal{S}_{R}(M)$. Hence $\delta\left(\mathcal{S}_{R}(M)\right) \geq \delta\left(\mathcal{P}_{R}(M)\right) \geq 1$. Moreover, if $M$ contains a proper essential submodule (i.e. $M$ is not a semisimple module), then $\Delta\left(\mathcal{S}_{R}(M)\right)=\left|\mathcal{V}\left(\mathcal{S}_{R}(M)\right)\right|-1$. The above remarks will be used freely in the text. Using the above it is easy to see that: 
Proposition 1.1. Suppose the module $M$ is not simple. Then $M$ is semisimple if and only if $\mathcal{S}_{R}(M)=\mathcal{P}_{R}(M)$ if and only if there exists a vertex $X$ of $\mathcal{P}_{R}(M)$, such that $\operatorname{deg}_{\mathcal{S}_{R}(M)}(X)=$ $\operatorname{deg}_{\mathcal{P}_{R}(M)}(X)$.

Example 1.2. Let $M=\bigoplus_{i \in T} B_{i}$ be a direct sum of pairwise non isomorphic simple $R$ modules, where $T$ is a finite set with $|T|=n \geq 2$. Then submodules of $M$ are in one to one correspondence with subsets of $T$. Let $A=\bigoplus_{i \in S} B_{i}$ and $B=\bigoplus_{i \in S^{\prime}} B_{i}$ be a proper submodules $M$ such that $A+B=M$. Then $S^{\prime}=(T \backslash S) \cup F$, where $F$ is a subset of $S$ different from $S$. This shows that $\operatorname{deg}_{\mathcal{S}_{R}(M)}(A)=2^{|S|}-1$. In particular, maximal submodules correspond to vertices of $\mathcal{S}_{R}(M)$ of degree $2^{n-1}-1=\Delta\left(\mathcal{S}_{R}(M)\right)$ and minimal submodules to vertices of degree $1=\delta\left(\mathcal{S}_{R}(M)\right)$.

Notice that if $X, A \in \mathcal{V}\left(\mathcal{P}_{R}(M)\right)$ and $X \subseteq A$, then $\operatorname{deg}_{\mathcal{P}_{R}(M)}(X) \leq \operatorname{deg}_{\mathcal{P}_{R}(M)}(A)$. The following example shows that this inequality can be sharp even when $A$ is a uniform submodule of $M$.

Example 1.3. Consider the $\mathbb{Z}$-module $M=\mathbb{Z}_{8} \oplus \mathbb{Z}_{2}$ and set $A=\mathbb{Z}(1,0)=\mathbb{Z}_{8}$ and $X=$ $4 \mathbb{Z}_{8} \subseteq A$. Then $A$ is uniform and one can check that $\operatorname{deg}_{\mathcal{P}_{\mathbb{Z}}(M)}(X)=2$ and $\operatorname{deg}_{\mathcal{P}_{\mathbb{Z}}(M)}(A)=3$.

Example 1.4. Let $M=B_{1} \oplus B_{2}$, where $B_{i}$ 's are simple $R$-modules. Then:

(i) $\mid \mathcal{V}\left(\mathcal{S}_{R}(M)|=| \operatorname{Hom}_{R}\left(B_{1}, B_{2}\right) \mid+1\right.$

(ii) $\mathcal{S}_{R}(M)=\mathcal{P}_{R}(M)$ is a complete graph of cardinality $\left|\operatorname{Hom}_{R}\left(B_{1}, B_{2}\right)\right|+1$.

Indeed (i) is a direct consequence of [8, Lemma 1.1] (or can be easily seen directly) and (ii) is a consequence of (i).

Remark that Examples 1.2 and 1.4 apply when ${ }_{R} M={ }_{R} R$ and $R$ is a finite product of division rings and $R$ is a two by two matrix ring over a division ring, respectively.

We close this section with the following observation:

Theorem 1.5. $\mathcal{S}_{R}(M)$ and $\mathcal{P}_{R}(M)$ are connected graphs of diameter not bigger than 3.

Proof. Suppose there are nontrivial submodules $L \neq K$ such that $L$ and $K$ are not adjacent. Let $C, D$ denote complements to $L+K$ and $L \cap K$ in $M$, respectively.

If $L \cap K=0$, then we have the path $L \backsim K \oplus C \backsim L \oplus C \backsim K$.

If $L \cap K \neq 0$, then we have the path $L \backsim D \backsim K$. Notice all submodules appearing above are not essential, so the thesis follows.

\section{On Vertices of FINITE DEGREe}

The aim of this section is to characterize modules $M$ such that all vertices of the proper sum-essential $\mathcal{P}_{R}(M)$ are of finite degree and to determine submodules of degree one. Let us begin the following theorem.

Theorem 2.1. For a module $M$ the following conditions are equivalent:

(1) $M$ has only finitely many submodules;

(2) $\Delta\left(\mathcal{S}_{R}(M)\right)$ is finite;

(3) Every vertex of $\mathcal{S}_{R}(M)$ is of finite degree;

(4) Either (i) $M$ contains a proper essential submodule $Q$ of finite degree in $\mathcal{S}_{R}(M)$ or (ii) There exist simple submodules $S_{1}, \ldots, S_{n}$ of $M$ such that $M=\bigoplus_{i=1}^{n} S_{i}$ and $\left|\operatorname{Hom}\left(S_{i}, S_{j}\right)\right|<\infty$, for all $1 \leq k<l \leq n$. 
Proof. Implications $(1) \Rightarrow(2) \Rightarrow(3)$ are trivial.

$(3) \Rightarrow(4)$. Suppose $M$ does not contain a proper essential submodule, i.e. $M$ is semisimple. Let $M=\bigoplus_{i \in I} S_{i}$ be a direct sum of simple modules $S_{i}$, where $i$ ranges over an index set $I$. Pick $k \in I$. Since, for any proper subset $J$ of $I \backslash\{k\}, \bigoplus_{k \neq i \in I} S_{i}$ is adjacent to $S_{k} \oplus \bigoplus_{i \in J} S_{i}$, the set $I \backslash\{k\}$ has finitely many subsets, so $I$ is finite, say $I=\{1, \ldots, n\}$.

Let us fix $1 \leq k<l \leq n$ and set $N=\bigoplus_{i \neq l}^{n} S_{i}$. Then, for any nonzero submodule $B$ of $S_{k} \oplus S_{l}$ different from $S_{k}$, we have $B+N=M$. Since $\operatorname{deg}_{\mathcal{S}_{R}(M)}(N)$ is finite, Example 1.4 shows that $\left|\operatorname{Hom}\left(S_{k}, S_{l}\right)\right|<\infty$ for all $1 \leq k<l \leq n$, i.e. (4)(ii) holds.

(4) $\Rightarrow(1)$. If $M$ is as in (4)(i), then $M$ has finitely many submodules. Suppose (4)(ii) holds and $M=\bigoplus_{i=1}^{n} S_{i}$. Since $M$ is semisimple, it is enough to see that $M$ has only finitely many simple submodules. Let $\mathcal{S}_{1}$ denote the set of all submodules isomorphic to $S_{1}$ and $\pi_{S, i}: S \rightarrow S_{i}$, where $S \in \mathcal{S}_{1}$ and $1 \leq i \leq n$. Set $I=\left\{i \mid \pi_{S, i} \neq 0\right\}$. If $|I|=1$, then $S=S_{1}$. Suppose $|I|>1$. Then $S_{i} \simeq S_{j}$, for $i, j \in I$ and any $S \in \mathcal{S}_{1}$ is a submodule of $V=\bigoplus_{i \in I} S_{i}$. By (4)(ii), $\left|\operatorname{End}\left(S_{i}\right)\right|<\infty$ so also $\operatorname{End}(V)$ is finite. This, in particular implies that $\mathcal{S}_{1}$ is finite. Replacing $S_{1}$ by any $S_{k}, 1 \leq k \leq n$, in the above consideration we see that $M$ has finitely many simple submodules and (1) follows.

For obtaining a similar result for the proper sum-essential graph we will need the following lemma:

Lemma 2.2. Suppose that $\operatorname{deg}_{\mathcal{P}_{R}(M)}(A)<\infty$, for any $A \in \mathcal{V}\left(\mathcal{P}_{R}(M)\right)$. Then $\operatorname{soc}(M) \leqslant_{e} M$ and $\operatorname{soc}(M)$ contains only finitely many submodules.

Proof. Suppose $\mathcal{P}_{R}(M)$ is not an empty graph. Let us observe first that udim( $(M)$ is finite. Indeed in case $M$ would contain nonzero submodules $A_{i}$ such that $\bigoplus_{i=1}^{\infty} A_{i} \leqslant e$, then $N=\bigoplus_{i=2}^{\infty} A_{i}$ would be adjacent to infinitely many vertices $A_{1} \oplus A_{k}$, for $k \in \mathbb{N}$. This is impossible as $\operatorname{deg} N<\infty$. Let $U$ be a uniform submodule of $M$ and $C$ a complement to $U$ in $M$. Then, for any nonzero submodule $A$ of $U, A \oplus C \leqslant_{e} M$, as $A \leqslant_{e} U$. This shows that every nonzero uniform submodule contains only finitely many submodules, in particular, it contains a simple submodule. Hence $\operatorname{soc}(M) \leqslant_{e} M$, as $\operatorname{udim}(M)<\infty$. This means that every vertex of the graph $\mathcal{P}_{R}(\operatorname{soc}(M))=\mathcal{S}_{R}(\operatorname{soc}(M))$ is of finite degree, as $\mathcal{P}_{R}(M)$ has this property. Now, applying Theorem 2.1 to $\mathcal{S}_{R}(\operatorname{soc}(M))$ we get $\left|\mathcal{P}_{R}(\operatorname{soc}(M))\right|<\infty$, i.e. the thesis holds.

Theorem 2.3. For a module $M$ the following conditions are equivalent:

(1) Every vertex of $\mathcal{P}_{R}(M)$ is of finite degree;

(2) The graph $\mathcal{P}_{R}(M)$ is finite.

Proof. (1) $\Rightarrow(2)$ In virtue of Lemma 2.2, it is enough to show that for every proper submodule $P$ of $\operatorname{soc}(M)$ the set $\mathcal{A}_{P}=\{N<M \mid N \cap \operatorname{soc}(M)=P\}$ is finite. Let $C_{P}$ be a complement to $P$ in $M$. Then $C_{P} \neq 0$ as $P$ is a proper submodule of $\operatorname{soc}(M)$. Moreover $C_{P}$ is adjacent to any $N \in \mathcal{A}_{P}$ and $\left|\mathcal{A}_{P}\right|<\infty$ follows, as $\operatorname{deg}_{\mathcal{P}_{R}(M)}\left(C_{P}\right)<\infty$.

The implication $(2) \Rightarrow(1)$ is clear.

Lemma 2.4. Let $B \in \mathcal{V}\left(\mathcal{S}_{R}(M)\right)$. If $\operatorname{deg}_{\mathcal{S}_{R}(M)}(B)=1$, then either $B$ is a simple module or there exists a simple submodule $A \subset B$ such that $\mathcal{V}\left(\mathcal{S}_{R}(M)\right)=\{A, B\}$.

Proof. Suppose $B$ is not simple and let $A$ be a nonzero proper submodule of $B$. If $B$ is essential in $M$, then clearly the second case holds. 
Thus we may assume that $B$ is not essential in $M$. Let $C$ be a complement to $B$. If $A \oplus C$ is a proper submodule of $M$, then $B$ is adjacent to $C$ and $A \oplus C$. This yields $A=0$, which is impossible. Thus $A \oplus C=M$. Then also $B \oplus C=M$ and $A=B$ follows, a contradiction. This shows that if $B$ is not essential, then it is simple.

Proposition 2.5. For a submodule $B$ of a semisimple module $M$ the following conditions are equivalent:

(1) $\operatorname{deg}_{\mathcal{S}_{R}(M)}(B)=1$;

(2) $B$ is simple and has unique nonzero complement in $M$;

(3) $B$ is simple, $M$ is not simple and has no other submodules isomorphic to $B$.

Proof. (1) $\Leftrightarrow(2)$ Suppose $\operatorname{deg}_{\mathcal{S}_{R}(M)}(B)=1$. Then, by Lemma 2.4, $B$ is simple. It is also clear that the complement to $B$ in $M$ is unique. The reverse implication is obvious.

$(2) \Rightarrow(3)$ Let $C$ be the unique complement to $B$. Let $D$ be any submodule of $M$ such that $B \cap D=0$ and $E$ a complement to $B \oplus D$ in $M$. Since $M$ is semisimple we get $B \oplus D \oplus E=M$ and $D \subseteq C$ follows as $C$ is unique. In case $D$ would be isomorphic to $B$ then both $D$ and the submodule $D^{\prime}=\{a+f(a) \mid a \in B\}$, where $f: B \rightarrow D$ is the isomorphism, would be contained in $C$ and $B \subseteq D+D^{\prime} \subseteq C$. This is impossible, so $M$ does not contain submodules isomorphic to $B$, i.e. the statement (3) holds.

$(3) \Rightarrow(2)$ Suppose $B$ is a submodule of $M$ satisfying (3). Let $P, Q \subseteq M$ be complements to $B$ in $M$ and let $\pi: M=B \oplus Q \rightarrow B$ denote the projection onto $B$ along $Q$. If $\pi(P) \neq 0$, then $\pi(P)=B$ as $B$ is simple. Then, as $P$ is semisimple, there exists a submodule $S \subseteq P$ such that $P=(\operatorname{ker} \pi \cap P) \oplus S$. Thus $S$ is isomorphic to $B$ and $B=S \subseteq P$ follows, which is impossible. Hence $\pi(P)=0$ and consequently $P \subseteq Q=\operatorname{ker} \pi$ and maximality of $P$ gives $P=Q$, i.e. (2) holds.

As a direct application of the above proposition and Lemma 2.4 we obtain the following corollaries:

Corollary 2.6. Let $R$ be a semisimple ring and $B$ a minimal left ideal of $R$. Then $\operatorname{deg}_{\mathcal{S}_{R}(R)}(B)=$ 1 if and only if $B$ is a two-sided ideal of $R$. In particular, every minimal left ideal of $R$ is of degree 1 in $\mathcal{S}_{R}(R)$ if and only if $R$ is a direct product of division rings.

Corollary 2.7. The sum-essential graph $\mathcal{S}_{R}(M)$ possesses a vertex of degree 1 if and only if either $M$ is a semisimple module possessing a proper simple submodule $B$ such that $M$ does not contain other submodules isomorphic to $B$ or $M$ is a chain module with the graph with two vertices (then any vertex is of degree 1 ).

Our next goal is to describe, in Theorem 2.13 modules $M$ such that $\mathcal{P}_{R}(M)$ contains a vertex of degree one. For this we need a series of lemmas.

Lemma 2.8. Let $U$ be a proper uniform submodule of $M$ and $S=U \cap \operatorname{soc}(M)$. Suppose that any submodule $D$ of $M$ satisfying $U \cap D=0$ is contained in $\operatorname{soc}(M)$. Then:

(1) $U+\operatorname{soc}(M) \leqslant e$;

(2) If $S=0$, then $U^{\prime}=\operatorname{soc}(M)$ is the unique complement to $U$ in $M$;

(3) If $S \neq 0$, then $S$ is simple and a submodule $C$ is a complement to $U$ in $M$ if and only if $C$ is the complement to $S$ in $\operatorname{soc}(M)$. In particular, if $C$ is a complement to $U$ in $M$, then $C \oplus S=\operatorname{soc}(M)$.

Proof. Let us notice that, due to our assumption, any complement $U^{\prime}$ of $U$ in $M$ is contained in the socle of $M$. In particular (1) holds. The above also implies (2). 
Suppose now that $S \neq 0$. Then $S$ is a simple module, as $U$ is uniform. Let $U^{\prime}$ be a complement to $U$ in $M$ and $W$ a complement to $S$ in $\operatorname{soc}(M)$. We know that $U^{\prime}$ is semisimple, so $U^{\prime}$ is also a complement of $S$ in $\operatorname{soc}(M)$ (as $S \oplus U^{\prime} \leqslant_{e} U \oplus U^{\prime} \leqslant_{e} M$ ). Moreover, if $W \subseteq U^{\prime}$, then $W=U^{\prime}$, i.e. $W$ is a complement to $U$ in $M$. This yields (3).

In the following lemma we collect basic properties of vertices of degree one in $\mathcal{P}_{R}(M)$.

Lemma 2.9. Let $U \in \mathcal{V}\left(\mathcal{P}_{R}(M)\right)$. If $\operatorname{deg}_{\mathcal{P}_{R}(M)}(U)=1$, then:

(1) $U$ is a uniform module;

(2) $\operatorname{deg}_{\mathcal{P}_{R}(M)}(B)=1$, for every vertex $B \subseteq U$;

(3) The complement to $U$ in $M$ is a semisimple module;

(4) Let $E$ be a submodule of $M$ such that $E \cap U \neq 0$ and $E+U \leqslant_{e} M$, then $\operatorname{soc}(M) \subseteq E$.

Proof. Suppose $\operatorname{deg}_{\mathcal{P}_{R}(M)}(U)=1$ and let $C$ be the complement to $U$ in $M$. Then, for any nonzero submodule $B$ of $U, U+(B+C) \leqslant_{e} M$. Since $\operatorname{deg}_{\mathcal{P}_{R}(M)}(U)=1$ and $C \neq B+C$, $B+C \leqslant_{e} M$ follows. This easily yields (1) and (2).

(3) If $X$ is a proper essential submodule of $C$, then $X \oplus U \leqslant_{e} M$, as $U \oplus X \leqslant_{e} U \oplus C \leqslant_{e} M$. Therefore, as $\operatorname{deg}_{\mathcal{P}_{R}(M)}(U)=1, C$ has no proper essential submodules so is semisimple and (3) holds.

(4) By (1), $U$ is uniform. Let $E$ be a submodule of $M$ satisfying assumptions of (4) and $S$ be a simple submodule of $M$. If $S \subseteq U$, then $S=S \cap(U \cap E) \subseteq E$.

Now suppose that $S \cap U=0$. If also $S \cap E=0$ then $E$ would not be essential in $M$ and $U$ would be adjacent to two different vertices, $E$ and the complement of $U$ which contains $S$. Hence $S \subseteq E$ and $\operatorname{soc}(M) \subseteq E$ follows.

Lemmas 2.8 and 2.9 give immediately the following

Corollary 2.10. Suppose $U \in \mathcal{V}\left(\mathcal{P}_{R}(M)\right)$ is a vertex of degree one. Let $C$ be the unique complement to $U$ in $M$ and $S=U \cap \operatorname{soc}(M)$. Then:

(1) If $U \cap E=0$, for a submodule $E$ of $M$, then $E \subseteq \operatorname{soc}(M)$;

(2) $C$ is the unique complement to $S$ in $\operatorname{soc}(M)$;

(3) $S=0$ iff $C=\operatorname{soc}(M)$ (in this case $\operatorname{udim}(M)=\operatorname{udim}(\operatorname{soc}(M))+1)$;

(4) $S \neq 0$ iff $C \neq \operatorname{soc}(M)$ (in this case $\operatorname{udim}(M)=\operatorname{udim}(\operatorname{soc}(M))$ );

Proof. (1) is a direct consequence of Lemma 2.9(3). This statement yields (2) if $S=0$. For $S \neq 0,(2)$ is just Lemma 2.8(3). Now it is easy to complete the proof of the corollary.

The statement (2) of Lemma 2.9 yields also:

Corollary 2.11. Suppose that the graph $\mathcal{P}_{R}(M)$ has only finitely many vertices of degree 1 , then every such vertex contains a simple submodule of $M$.

In the sequel we will need yet another lemma.

Lemma 2.12. Suppose $U$ is a nontrivial uniform submodule of a left $R$-module $M$ with a nonzero complement $C$, where $C \subseteq \operatorname{soc}(M)$. Suppose there exist a simple submodule $F \nsubseteq U$, $0 \neq f \in F$ and $0 \neq u \in U$ such that for any $r \in R$, if $r u=0$ then $r f=0$. Then $\operatorname{deg}_{\mathcal{P}_{R}(M)}(U)>1$.

Proof. If $U$ has two different complements, then the result is clear. Thus let us assume that $C$ is the unique complement to $U$ in $M$. Since $U$ is uniform, $R u \oplus C \leqslant_{e} U \oplus C \leqslant_{e} M$ and $R u+C \leqslant_{e} M$ follows. 
Let $u, f \in M$ be as in the formulation of the lemma and $r, s \in R$. Notice that if $r u=s u$ then $(r-s) u=0$, so also $(r-s) f=0$, i.e. $r(u+f)=s(u+f)$. This shows that the map $\varphi: R u \rightarrow R(u+f)$ defined by $\varphi(r u)=r(u+f)$ is a well-defined epimorphism of $R$-modules. Moreover, since $r u \in \operatorname{ker} \varphi$ gives $r u=-r f \in U \cap F=0, \varphi$ is an isomorphism. Observe that $0 \neq f \in R(u+f)+R u$ and hence, as $F$ is simple, we have $R(u+f)+R u=R u \oplus F$. Thus $R(u+f)+W+R u \leqslant e M$, where $C=W \oplus F$. Notice that $R(u+f)+W$ is not essential in $M$. Otherwise, $F \subseteq R(u+f)+W$ and then $f=r u+r f-w$, for some $r \in R$ and $w \in W$. So $r u=(f-r f)+w \in C \cap R u=0$. Thus $r u=0$ and by assumption $r f=0$ follows. Now, from $f=r u+r f-w$, we get $f=-w \in W$, a contradiction. Therefore $R(u+f)+W$ is not essential in $M$ and the essentiality of $R(u+f)+W+R u$ in $M$ shows that $U$ is adjacent with

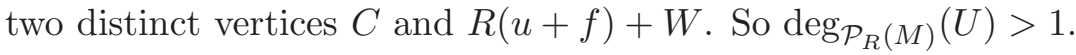

Now we are ready to prove the following theorem:

Theorem 2.13. Let $M$ a left $R$-module and $U \in \mathcal{V}\left(\mathcal{P}_{R}(M)\right)$.

The following conditions are equivalent:

(1) $\operatorname{deg}_{\mathcal{P}_{R}(M)}(U)=1$;

(2) (i) $U$ is uniform and the complement to $U$ in $M$ is unique and is semisimple;

(ii) For every simple submodule $F \nsubseteq U, 0 \neq f \in F$ and $0 \neq u \in U$ there exists an element $r \in R$ such that $r u=0$ and $r f \neq 0$;

(3) (i) $U$ is uniform and the complement to $U$ in $M$ is unique and is semisimple;

(ii) If $U \cap E \neq 0$ and $U+E \leqslant e$, for a submodule $E$ of $M$, then $\operatorname{soc}(M) \subseteq E$;

(4) For any submodule $E$ of $M$ the following conditions hold:

(i) If $U \cap E=0$ then $E \subseteq \operatorname{soc}(M)$;

(ii) $U \cap E \neq 0$ and $U+E \leq_{e} M$, then $\operatorname{soc}(M) \subseteq E$;

(iii) $U$ is uniform and $U \cap \operatorname{soc}(M)$ has unique complement in $\operatorname{soc}(M)$.

Proof. (1) $\Rightarrow(2)$ Suppose $\operatorname{deg}_{\mathcal{P}_{R}(M)}(U)=1$. Then part (i) holds by Corollary 2.10 and part (ii) is a direct consequence of Lemma 2.12 .

(2) $\Rightarrow(3)$ Let $F$ be a simple submodule of $M$. If $F \nsubseteq U$, then the condition (2)(ii) implies that $R(u+f) \cap F \neq 0$, for any $0 \neq u \in U$ and $0 \neq f \in F$. Hence $F \subseteq R(u+f)$. This shows that $R(u+f)=R u \oplus F$, for every simple submodule $F \nsubseteq U, 0 \neq f \in F$ and $u \in U$.

Let $E$ be a submodule of $M$ such that $E+U \leqslant_{e} M$ and $U \cap E \neq 0$. If $F \subseteq U$ then, as $U$ is uniform, $F \subseteq U \cap E$, so $F \subseteq E$. Suppose $F \nsubseteq \amalg$ and pick $0 \neq f \in F$. Then $f=u+e$, for some suitable $u \in U$ and $e \in E$. If $u=0$ then $f=e \in E$ and $F \subseteq E$ follows. If $u \neq 0$, then $E \supseteq R(-u+f)=R u \oplus F$. Thus in any case $F \subseteq E$ and (ii) follows.

$(3) \Rightarrow(1)$ Let $C$ be a complement to $U$ in $M$. By (3)(i), $C$ is unique and semisimple. Let $E$ be a submodule of $M$ such that $E+U \leqslant_{e} M$. If $E \subseteq C$, then $E=C$ as $C$ is semisimple. Thus, to prove the implication, it is enough to show that if $E \nsubseteq C$, then $E$ has to be essential in $M$. To this end, suppose $E \nsubseteq C$. Then, as the complement $C$ is unique, $E \cap U \neq 0$. Therefore, by (3)(ii), $\operatorname{soc}(M) \subseteq E$. Notice also that the condition (3)(i) guarantees that $U$ satisfies assumptions of Lemma 2.8, Let $S=U \cap \operatorname{soc}(M)$.

If $S=0$ (i.e. $C=\operatorname{soc}(M))$. Then the $\operatorname{sum}(U \cap E)+\operatorname{soc}(M)$ is direct. By (i), $U$ is uniform, hence $(U \cap E) \oplus \operatorname{soc}(M) \leqslant_{e} U \oplus \operatorname{soc}(M) \leqslant_{e} M$. This implies $E \leqslant_{e} M$, as $(U \cap E) \oplus \operatorname{soc}(M) \subseteq E$.

If $S \neq 0$ then Lemma 2.8(3) implies that $S$ simple and $C$ is the unique complement to $S$ in $\operatorname{soc}(M)$. Then $\operatorname{soc}(M)=S \oplus C \leqslant_{e} U \oplus C \leqslant_{e} M$ and $E \leqslant_{e} M$ follows, as $\operatorname{soc}(M) \subseteq E$. This completes the proof of (1). 
We have already noticed that (3)(i) implies that $U$ satisfies the assumptions of Lemma 2.8. Then the equivalence $(3) \Leftrightarrow(4)$ is a direct consequence of this lemma.

Remark 2.14. Using Proposition 2.5, one can see that the condition (4)(iii) in the above theorem is equivalent to

(iii') $U$ is uniform and $M$ has no other submodules isomorphic to $U \cap \operatorname{soc}(M)$.

The above remark together with Theorem 2.13 and Proposition 2.5 yield:

Remark 2.15. Suppose $\operatorname{deg}_{\mathcal{P}_{R}(M)}(U)=1$. Then $U$ is only adjacent to $C=\sum_{F \text { simple } F \neq S} F$, where $S=U \cap \operatorname{soc}(M)$.

Of course it may happen that the complement to a uniform nonessential submodule $U$ of $M$ is unique but $\operatorname{deg}_{\mathcal{P}_{R}(M)}(U)>1$. Indeed, let $R=\mathbb{Z}$ and $M=\mathbb{Z} \oplus \mathbb{Z}_{2}$. Then $R(1,0)$ has unique complement (equal to $R(0,1)=\operatorname{soc}(M)$ ) but $R(1,0)$ is adjoint to any submodule $R(k, 1), k \in \mathbb{N}$. Thus $\operatorname{deg}_{\mathcal{P}_{R}(M)}(R(1,0))=\infty$.

The following example and proposition offer various possible interrelations between submodules of degree one in a module $M$.

Example 2.16. (1) Let $R=\mathbb{Z} \oplus \mathbb{Z}_{2}, M={ }_{R} R$ and $U \in \mathcal{P}_{R}(M)$. Then $\operatorname{deg}_{\mathcal{P}_{R}(M)}(U)=1$ if and only if $U \subseteq R(1,0)=\mathbb{Z}$. In this case $U \cap \operatorname{soc}(M)=0$.

(2) Let $p, q \in \mathbb{N}$ be prime numbers, $R=\mathbb{Z}$ and $M=\mathbb{Z}_{p \infty} \oplus \mathbb{Z}_{q}$, where $\mathbb{Z}_{p \infty}=\mathbb{Z}\left[\frac{1}{p}\right] / \mathbb{Z}$ is the $p$-quasicyclic group. Then $\operatorname{soc}(M)=\mathbb{Z}_{p} \oplus \mathbb{Z}_{q}$. If $p \neq q$, then $\operatorname{deg}_{\mathcal{P}_{R}(M)}\left(\mathbb{Z}_{q}\right)=\infty$ and a submodule $U \in \mathcal{P}_{R}(M)$ is of degree 1 if and only if $U \subseteq \mathbb{Z}_{p \infty}$. If $p=q$, then $\mathcal{P}_{R}(M)$ has no vertices of degree one.

(3) Let $M$ be the $\mathbb{Z}$-module $\mathbb{Z}_{4} \oplus \mathbb{Z}_{3}$. Then $\operatorname{soc}(M)=2 \mathbb{Z}_{4} \oplus \mathbb{Z}_{3}, \mathbb{Z}_{4}$ and $2 \mathbb{Z}_{4}$ are the only submodules of degree one, while $\operatorname{deg}_{\mathcal{P}_{\mathbb{Z}}(M)}\left(\mathbb{Z}_{3}\right)=2$.

(4) Let $P \subseteq \mathbb{N}$ denote the set of primes. Then any simple submodule of the $\mathbb{Z}$-module $\bigoplus_{p \in P} \mathbb{Z}_{p}$ is of degree 1 .

Proposition 2.17. Suppose there exist two distinct submodules $A$ and $B$ of a module $M$ such that $\operatorname{deg}_{\mathcal{P}_{R}(M)}(A)=\operatorname{deg}_{\mathcal{P}_{R}(M)}(B)=1$. Then:

(1) If $A \cap B=0$ then $A$ and $B$ are simple non isomorphic modules;

(2) If $A \cap B \neq 0$ then $\operatorname{deg}_{\mathcal{P}_{R}(M)}(A+B)=1$;

(3) If $A+B \leqslant_{e} M$ then $A, B$ are simple non isomorphic and $\operatorname{soc}(M)=A+B$.

Proof. (1) Suppose $A \cap B=0$. Then, by Theorem 2.13(4) applied to $U=A$ and $U=B$, we see that $A$ and $B$ are simple modules. Moreover, due to Remark 2.14, $A \nsucceq B$.

(3) is an immediate consequence of Remark 2.15.

(2) Suppose $A \cap B \neq 0$. If $A$ and $B$ are simple, the thesis is clear. Suppose $B$ is not simple and let $S=A \cap \operatorname{soc}(M)=B \cap \operatorname{soc}(M)$. Then, by (3) $A+B$ is not essential in $M$. Moreover $A, B$ and $A \cap B$ have the same unique complement $C$ described in Remark 2.15,

Let $D$ be a vertex of $\mathcal{P}_{R}(M)$, which is adjacent to $A+B$. Applying Theorem 2.13 to $U=A$ and $E=B+D$ we obtain $B+\operatorname{soc}(M) \subseteq E=B+D$. Hence, as $B+\operatorname{soc}(M) \leqslant_{e} M$, also $B+D \leqslant_{e} M$ and $\operatorname{deg}_{\mathcal{P}_{R}(M)}(B)=1$ gives $D=C$, i.e. $\operatorname{deg}(A+B)=1$.

As we have seen earlier, submodules of $M$ which are of degree one as elements of $\mathcal{P}_{R}(M)$ are uniform. By the above lemma, if $A$ and $B$ are such submodules having nonzero intersection then $A+B$ is a uniform submodule of $M$. Notice that, in general, a sum of two uniform submodules having nonzero intersection is not always uniform.

We close this section with the following theorem: 
Theorem 2.18. Let $M$ be a module containing a submodule $A$ of degree 1 in $\mathcal{P}_{R}(M)$. Then either all submodules of degree 1 are simple or $M$ contains the unique largest submodule of degree 1.

Proof. Suppose $M$ contains a not simple submodule $A$ such that $\operatorname{deg}_{\mathcal{P}_{R}(M)}(A)=1$. Let $B \in \mathcal{V}\left(\mathcal{P}_{R}(M)\right)$ with $\operatorname{deg}_{\mathcal{P}_{R}(M)}(B)=1$. Then, by Proposition 2.17, we know that $A \cap B \neq 0$ and $\operatorname{deg}_{\mathcal{P}_{R}(M)}(A+B)=1$. Let $S=A \cap \operatorname{soc}(M)$ and $C$ be the complement to $S$ in $\operatorname{soc}(M)$. Then, by Remark 2.15, $C=\sum_{F \text { simple } F \neq S} F$ is the unique complement to $B$ in $M$.

Let $U$ be a uniform submodule such that $B \subseteq U$. We claim $C$ is a complement to $U$ in $M$. Clearly $U+C \leqslant_{e} M$. If $U \cap C \neq 0$, then there exists a simple module $F \subseteq C$ such that $F \subseteq U$. Then $F \subseteq B \cap \operatorname{soc}(M)=S$, as $U$ is uniform, a contradiction. This shows that $U \cap C=0$. Notice also that if $U \oplus D \leqslant \leqslant_{e} M$ then $B \cap D=0$ and $D \subseteq C$ follows, as $\operatorname{deg}_{\mathcal{P}_{R}(M)}(B)=1$. This proves the claim.

Let $\mathcal{A}=\left\{B \in \mathcal{V}\left(\mathcal{P}_{R}(M)\right) \mid \operatorname{deg}_{\mathcal{P}_{R}(M)}(B)=1\right.$ and $\left.A \subseteq B\right\}$, Notice that to prove the theorem it is enough to show only that every chain in $\mathcal{A}$ is bounded, then the thesis is a consequence of Zorn's Lemma and Proposition 2.17(2). To this end, let $\left\{U_{i}\right\}$ be a chain in $\mathcal{A}$ and $U=\bigcup_{i} U_{i}$. Then $U$ is uniform and the above shows that $C$ is the unique complement to $U$ in $M$. Let $F$ be a simple submodule of $M$ such that $F \nsubseteq U$. Let $0 \neq f \in F$ and $0 \neq u \in U$. Then $u \in U_{i}$, for some index $i$. Since $\operatorname{deg}_{\mathcal{P}_{R}(M)}\left(U_{i}\right)=1$ we may apply Theorem 2.13)(2)(ii) to find $r \in R$ such that $r u=0$ and $r f \neq 0$. The above shows that $U$ satisfies Theorem 2.13(2) and proves that $\operatorname{deg}_{\mathcal{P}_{R}(M)}(U)=1$, i.e. $U \in \mathcal{A}$. This yields the thesis.

Let us notice that in case $\mathcal{P}_{R}(M)$ is a finite graph, the above theorem is a direct consequence of Proposition 2.17.

\section{Complete, $k$-regular and triangle-free graphs $\mathcal{S}_{R}(M)$ and $\mathcal{P}_{R}(M)$}

Let us begin this section with the following easy observation:

Lemma 3.1. Let $A \in \mathcal{V}\left(\mathcal{S}_{R}(M)\right)$ be a universal vertex. If $A \mathbb{K}_{e} M$, then $A$ is a simple module.

Proof. Notice that if $A \Varangle_{e} M$ then $A$ can not be adjacent to any of its submodules. Since $A$ is a universal vertex, the thesis follows.

The following theorem gives a characterization of modules with a complete sum-essential graph.

Theorem 3.2. Let $M$ be a nonzero nonsimple module. Then, $\mathcal{S}_{R}(M)$ is a complete graph if and only if one of the following conditions hold:

(1) $M$ is a uniform module;

(2) (i) Every nonzero nonessential submodule of $M$ is simple;

(ii) $\operatorname{soc}(M)=S_{1} \oplus S_{2} \leqslant_{e} M$, where $S_{1}, S_{2}$ are simple submodules.

In particular, if $M$ is semisimple, then $\mathcal{S}_{R}(M)$ is a complete graph if and only if $M$ is direct sum of two simple modules.

Proof. Suppose $M$ is not uniform. Then Lemma 3.1 shows that (2)(i) holds. Now (2)(ii) is a consequence of (i) and the assumption that every vertex is universal. 
Let us notice that every universal vertex of $\mathcal{P}_{R}(M)$ is also universal in $\mathcal{S}_{R}(M)$ and hence a nonempty graph $\mathcal{P}_{R}(M)$ is complete if and only if $\mathcal{S}_{R}(M)$ is such. Thus the above theorem gives the following corollary:

Corollary 3.3. Suppose $M$ is not uniform. Then, $\mathcal{P}_{R}(M)$ is a complete graph if and only if the following conditions hold:

(i) Every nonzero nonessential submodule of $M$ is simple;

(ii) $\operatorname{soc}(M)=S_{1} \oplus S_{2} \leqslant_{e} M$, where $S_{1}, S_{2}$ are simple submodules.

If $M$ does not contain proper essential submodules (i.e. if $M$ is semisimple) something more can be said. The last statement of the following corollary is a consequence of Example 1.4

Corollary 3.4. For a semisimple module $M$, the following conditions are equivalent:

(1) $\mathcal{P}_{R}(M)$ is a complete graph;

(2) $\mathcal{P}_{R}(M)$ contains a universal vertex;

(3) $M=S_{1} \oplus S_{2}$ for some simple modules $S_{1}$ and $S_{2}$.

In this case $\mathcal{S}_{R}(M)$ is a complete graph and $\left|\mathcal{V}\left(\mathcal{P}_{R}(M)\right)\right|=\left|\mathcal{V}\left(\mathcal{S}_{R}(M)\right)\right|=\left|\operatorname{Hom}\left(S_{1}, S_{2}\right)\right|+1$.

Corollary 3.5. Let $R$ be a semisimple ring with a nontrivial left ideal. Then $\mathcal{S}_{R}(R)$ is a complete graph if and only if $R \cong M_{2}(D)$ or $R \cong D_{1} \times D_{2}$, where $D, D_{1}$ and $D_{2}$ are division rings.

In Theorem 3.2 a characterization of modules with a complete sum-essential graph was given. The following theorem shows that $k$-regular sum-essential graph have to be complete.

Theorem 3.6. Let $M$ be a nonzero nonsimple module and $k \in \mathbb{N}$. The following conditions are equivalent:

(1) The graph $\mathcal{S}_{R}(M)$ is k-regular;

(2) $\mathcal{S}_{R}(M)$ is a complete graph and $\left|\mathcal{V}\left(\mathcal{S}_{R}(M)\right)\right|=k+1$.

Proof. $(1) \Rightarrow(2)$ Let $\mathcal{S}_{R}(M)$ be a $k$-regular. Suppose $M$ contains a proper essential submodule $E$. Then $E$ is adjacent to any $A \in \mathcal{V}\left(\mathcal{S}_{R}(M)\right) \backslash\{E\}$. Then $\left|\mathcal{V}\left(\mathcal{S}_{R}(M)\right)\right|=k+1$ and $\mathcal{V}(M)$ is a complete graph.

Assume now that $M$ does not have proper essential submodule, i.e. $M$ is semisimple. Let $S$ be a simple submodule of $M$. Then $k$ is the number of maximal submodules of $M$ not containing $S$. Let $N_{1}, \ldots, N_{k}$ be such maximal submodules. Then $N_{1}$ is adjacent to any $N_{i}$, $1<i \leq k$ and to arbitrary proper submodule of $M$ containing $S$. The fact that $\mathcal{S}_{R}(M)$ is $k$-regular implies that $M$ has no proper submodules containing $S$. Thus the semisimple module $M=S \oplus Q$ for suitable simple submodule $Q$ of $M$. Now the result is a consequence of Example 1.4.

The implication $(2) \Rightarrow(1)$ is clear.

The following theorem presents the structure of modules having triangle-free proper sumessential graphs.

Theorem 3.7. Let $M$ be a module such that $\left|\mathcal{V}\left(\mathcal{S}_{R}(M)\right)\right| \geq 2$. The following conditions are equivalent:

(1) The graph $\mathcal{S}_{R}(M)$ is triangle-free;

(2) Either $M$ is a direct sum of two non-isomorphic simple modules or $M$ is a chain module with exactly two proper submodules. 
(3) $\mathcal{S}_{R}(M) \simeq \mathcal{K}_{2}$ the complete graph with two vertices.

Proof. (1) $\Rightarrow(2)$ Suppose $\mathcal{S}_{R}(M)$ is a triangle-free. If $M$ would contain an essential direct sum $P \oplus Q \oplus S$ of nonzero submodules, then $P \oplus Q, P \oplus S, Q \oplus S$ would form a triangle, a contradiction. Hence udim $(M) \leq 2$.

If $\operatorname{udim}(M)=1$, then any two vertices are adjacent, so $M$ has to be a chain module with two vertices only.

Suppose udim $(M)=2$ and let $P$ be a uniform submodule and $Q$ its complement in $M$. If $P$ would contain a proper nonzero submodule $S$ then $S \oplus Q, P$ and $Q$ would form a triangle. Thus $P$ and $Q$ are simple and $P \oplus Q \leqslant e$. If $P \oplus Q$ would be a proper submodule, then we would have a triangle with vertices $P, Q, P \oplus Q$. Therefore $M=P \oplus Q$ in this case. Moreover $P$ and $Q$ are not isomorphic, as three distinct simple submodules would form a triangle. This completes the proof.

Implications $(2) \Rightarrow(3) \Rightarrow(1)$ are clear.

Corollary 3.8. Let $M$ be a nonzero nonsimple module. Then the girth of the graph $\mathcal{S}_{R}(M)$ is either 3 or $\infty$.

Proof. If $\mathcal{S}_{R}(M)$ contains a triangle then $g\left(\mathcal{S}_{R}(M)\right)=3$. If $\mathcal{S}_{R}(M)$ is triangle-free then, by Theorem [3.7, $\mathcal{S}_{R}(M)=\mathcal{K}_{2}$ or $\mathcal{S}_{R}(M)$ is a single point and $g\left(\mathcal{S}_{R}(M)\right)=\infty$.

Remark that bipartite graphs are triangle-free, so the above corollary applies in this case.

Definition 3.9. We say that submodules $A$ and $B$ of a module $M$ are strongly disjoint if there are no nonzero isomorphic submodules $X, Y$ of $M$ such that $X \subseteq A$ and $Y \subseteq B$.

The following lemma offers some other characterizations of strongly disjoint submodules.

Lemma 3.10. For submodules $A$ and $B$ of a left $R$-module $M$ the following conditions are equivalent:

(1) $A$ and $B$ are strongly disjoint;

(2) For any nonzero elements $a \in A$ and $b \in B$, $\operatorname{ann}(a) \neq \operatorname{ann}(b)$;

(3) (i) $A \cap B=0$;

(ii) If $U$ is a nonzero submodule of $A+B$, then $U \cap A \neq 0$ or $U \cap B \neq 0$.

Proof. The implication (1) $\Rightarrow(2)$ is clear as any cyclic $R$-module $R c$ isomorphic to $R / \operatorname{ann}(c)$.

$(2) \Rightarrow(3)$ Suppose (2) holds. Then clearly $A \cap B=0$. Let $U$ be a nonzero submodule of $A+B$ and $0 \neq u=a+b$, with $a \in A$ and $b \in B$. If one of $a$ and $b$ is equal to zero, then the result holds. If both elements are nonzero we can use (2) to find an element $r \in R$ which belongs to exactly one of the sets $\operatorname{ann}(a)$ and $\operatorname{ann}(b)$. Then either $r u=r b \neq 0$ or $r u=r a \neq 0$ and the result follows.

(3) $\Rightarrow$ (1) Let $X \subseteq A$ and $Y \subseteq B$ be isomorphic submodules. Let us consider the submodule $U=\{x+\phi(x) \mid x \in X\} \subseteq A+B$, where $\phi: X \rightarrow Y$ is a given isomorphism. Let $u \in U$ and $x \in X$ be such that $u=x+\phi(x)$. If $u \in U \cap A$, then $\phi(x)=u-x \in A \cap B=0$ and $U \cap A=0$ follows. Similarly, if $u \in U \cap B$, then $x=u-\phi(x) \in A \cap B=0$ yields $U \cap B=0$. Therefore, by (3), $X \simeq U=0$, i.e. $A$ and $B$ are strongly disjoint.

Theorem 3.11. Let $M$ be a module with nonempty graph $\mathcal{P}_{R}(M)$. The following conditions are equivalent:

(1) $\mathcal{P}_{R}(M)$ is triangle-free;

(2) (i) $\operatorname{udim}(M)=2$ (so any nonzero, nonessential submodule is uniform); 
(ii) If $A, B \in \mathcal{V}\left(\mathcal{P}_{R}(M)\right)$ and $A+B \leqslant_{e} M$, then $A$ and $B$ are strongly disjoint.

(3) If $A, B \in \mathcal{V}\left(\mathcal{P}_{R}(M)\right)$ and $A+B \leqslant_{e} M$, then $A$ and $B$ are strongly disjoint.

Proof. (1) $\Rightarrow(2)$ Assume $\mathcal{P}_{R}(M)$ is triangle-free. By assumption $M$ is not uniform. The argument used at the beginning of the proof of Theorem 3.7 shows that $\operatorname{udim}(M) \leq 2$, i.e. (2) $(i)$ holds.

Let $A, B \in \mathcal{V}\left(\mathcal{P}_{R}(M)\right)$ and $A+B \leqslant_{e} M$. Let $N=A \cap B$ and $N^{\prime}$ be the complement to $N$ in $M$. If $N$ would be nonzero, then $N^{\prime}$ would be a proper nonessential submodule of $M$ and we would have a triangle with vertices $A, B, N^{\prime}$ (as $A+B \leqslant_{e} M$ ). This is impossible, so $A \cap B=0$. Let $U$ be a uniform nonzero submodule of $A+B$. Using (2)(i) we see that $A \cap U \neq 0$ or $B \cap U \neq 0$, as otherwise $A, B, U$ would form a triangle. Now (2)(ii) is a consequence of Lemma 3.10 .

The implication $(2) \Rightarrow(3)$ is clear.

(3) $\Rightarrow(1)$ Let $A, B, C \in \mathcal{V}\left(\mathcal{P}_{R}(M)\right)$ be such that $A+C \leqslant_{e} M$ and $B+C \leqslant_{e} M$. In particular, by assumption, we have $A \cap C=0=B \cap C$. Let $U=C \cap(A+B)$. Notice that $A+B \Varangle_{e} M$, as otherwise $U \neq 0$ and (3) would imply that either $U \cap A \neq 0$ or $U \cap B \neq 0$, which is impossible. This shows that $\mathcal{P}_{R}(M)$ is triangle free.

Theorem 3.12. Let $M$ be a module with $\mathcal{V}\left(\mathcal{P}_{R}(M)\right) \neq \emptyset$. Then the following conditions are equivalent:

(1) $\mathcal{P}_{R}(M)$ is a tree;

(2) If $A, B \in \mathcal{V}\left(\mathcal{P}_{R}(M)\right)$ and $A+B \leqslant_{e} M$, then $A$ and $B$ are strongly disjoint and one of $A$ and $B$ is a simple module;

(3) $\mathcal{P}_{R}(M)$ is a star graph with the center $S$, for a simple submodule $S$ of $M$.

Proof. (1) $\Rightarrow(2)$ Every tree is triangle-free, so the first part of the statement (2) is given by Theorem 3.11. Let $A, B \in \mathcal{V}\left(\mathcal{P}_{R}(M)\right)$ be such that $A+B \leqslant_{e} M$. In case we would have proper nonzero submodules $X$ and $Y$ of $A$ and $B$, respectively, we would have a cycle $X \backsim B \backsim A \backsim Y \backsim X$ in a tree. Hence one of $A, B$ has to be a simple module, i.e. (2) holds.

$(2) \Rightarrow(3)$ Suppose (2) holds. Then $M$ contains a simple submodule, call it $S$. Theorem 3.11 shows that $\mathcal{P}_{R}(M)$ is triangle free and $\operatorname{udim}(M)=2$. In particular $M$ has at most two different simple submodules.

If $\operatorname{soc}(M)=S$ then, by (2), $\mathcal{P}_{R}(M)$ is a star graph with center $S$.

Suppose $\operatorname{soc}(M)$ is not simple. Since $\operatorname{udim}(M)=2, S \oplus Q=\operatorname{soc}(M) \leqslant_{e} M$ where $S, Q$ are the only simple submodules of $M$. In particular, any $A \in \mathcal{V}\left(\mathcal{P}_{R}(M)\right)$ contains one of $S$ or $Q$. The statement (2) implies that one of the simples, say $S$, does not have proper essential extensions in $M$. Therefore, if $S \neq A \in \mathcal{V}\left(\mathcal{P}_{R}(M)\right)$, then $Q \subseteq A$ and $\mathcal{P}_{R}(M)$ is a star graph with center $S$.

The implication $(3) \Rightarrow(1)$ is clear.

Let us remark that the proof of the implication $(2) \Rightarrow(3)$ gives the module structure of modules with $\mathcal{P}_{R}(M)$ being a tree. The $\mathbb{Z}$-modules $\mathbb{Z} \oplus \mathbb{Z}_{q}$ or $\mathbb{Z}_{p^{n}} \oplus \mathbb{Z}_{q}$ are examples of such modules, where $p, q, n \in \mathbb{N}$ and $p \neq q$ are prime. We have seen in Corollary 3.8 that the girth of $\mathcal{S}_{R}(M)$ is equal to 3 when finite. In case of the graph $\mathcal{P}_{R}(M)$ we have the following:

Corollary 3.13. Suppose $\mathcal{P}_{R}(M)$ is a nonempty graph. Then $g\left(\mathcal{P}_{R}(M)\right) \in\{3,4, \infty\}$.

Proof. Assume $\mathcal{P}_{R}(M)$ is a triangle-free graph, i.e. $g\left(\mathcal{P}_{R}(M)\right) \neq 3$. If $\mathcal{P}_{R}(M)$ is not a tree then, using Theorems 3.12 and 3.11 we can pick two strongly disjoint uniform submodules $A$ and $B$ in $\mathcal{P}_{R}(M)$ such that $A+B \leqslant_{e} M$ and neither $A$ nor $B$ is simple. Let $X, Y$ 
be proper nonzero submodules of $A$ and $B$, respectively. Then $X, Y, A, B$ form a cycle, so $g\left(\mathcal{P}_{R}(M)\right)=4$. This yields the thesis.

The girth of $\mathcal{P}_{R}(M)$ can take any value as described above. If udim $(M)>2$, then $g\left(\mathcal{P}_{R}(M)\right)=3$. If $\operatorname{udim}(M)=2$ consider the group $M=\mathbb{Z}_{p^{m}} \oplus \mathbb{Z}_{q^{n}}$ as a $\mathbb{Z}$-module, where $p, q \in \mathbb{N}$ are different primes and $n, m \geq 1$. Then any nonessential submodule of $M$ is contained either in $\mathbb{Z}_{p^{m}}$ or $\mathbb{Z}_{q^{n}}$ and Theorem 3.11 (or direct argument) shows that $\mathcal{P}_{\mathbb{Z}}(M)$ is triangle free. If $n=1$, then $g\left(\mathcal{P}_{\mathbb{Z}}(M)\right)=\infty$. If $n, m \geq 2$, then $g\left(\mathcal{P}_{\mathbb{Z}}(M)\right)=4$.

We close the paper with the following result:

Proposition 3.14. Suppose that the module $M$ has exactly $2 \leq n<\infty$ maximal submodules. The following conditions are equivalent:

(1) $\mathcal{S}_{R}(M)$ is n-partite graph;

(2) $M$ is a semisimple module.

Proof. Let $M_{1}, \ldots, M_{n}$ be the maximal submodules of $M, J=J(M)=\bigcap_{i=1}^{n} M_{i}$ and $N$ be the complement to $J$ in $M$.

$(1) \Rightarrow(2)$ In case $J$ would be nonzero then $N, M_{1}, \ldots, M_{n}$ would generate a complete subgraph of $\mathcal{S}_{R}(M)$. Since $\mathcal{S}_{R}(M)$ is $n$-partite graph, this is impossible and $J=0$ follows. This yields the thesis.

$(2) \Rightarrow(1)$ Suppose $M$ is a semisimple module. For $1 \leq k \leq n$, let us define $V_{k}=\{A \in$ $\mathcal{V}\left(\mathcal{S}_{R}(M)\right) \mid A \subseteq M_{k}$ and $A \nsubseteq M_{i}$ for $\left.i<k\right\}$. Then, using semisimplicity of $M$ it is easy to see that $V_{1}, \ldots, V_{n}$ form $n$-partitioning subsets of $\mathcal{V}\left(\mathcal{S}_{R}(M)\right)$.

\section{REFERENCES}

[1] S. Akbari, M. Habibi, A. Majidinya, R. Manaviyat, The inclusion ideal graph of rings, Comm. Algebra 43(6) (2015)2457-2465, DOI: 10.1080/00927872.2014.894051.

[2] S. Akbari, R. Nikandish and J. Nikmehr, Some results on the intersection graphs of ideals of rings, J. Algebra Appl. 12 (2013) 1250200, DOI: 10.1142/S0219498812502003.

[3] S. Akbari, H. A. Tavallaee and S. Khakashi Ghezelahmad, Intersection graph of submodules of a module, J. Algebra Appl. 11 (2012) 1250019, DOI: 10.1142/S0219498811005452.

[4] A. Amini, B. Amini, E. Momtahan, M.H. Shirdareh, Haghighi, On a graph of ideals, Acta Math. Hung. 134 (2012) 369-384, DOI: 10.1007/s10474-011-0121-3.

[5] J. Amjadi, The essential ideal graph of a commutative ring, Asian-Eur. J. Math. Vol. 11, No. 2 (2018)1850058. DOI: 10.1142/S1793557118500584.

[6] T.Y. Lam, A First Course in Noncommutative Rings, Springer-Verlag, New York, 2001.

[7] T.Y. Lam, Lectures on Modules and Rings, Springer-Verlag, New York 1999.

[8] J. Matczuk, M. Nowakowska, E. R Puczyłowski, Intersection graphs of modules and rings, J. Algebra Appl. 17(3) (2018) 1850131, DOI: 10.1142/S0219498818501311.

[9] H. J. Wang, Graphs associated to co-maximal ideals of commutative rings, J. Algebra 320 (2008) 29172933, DOI: 10.1016/j.jalgebra.2008.06.020.

[10] H. J. Wang, Co-maximal graph of non-commutative rings, Linear Algebra Appl. 430 (2009) 633-641, DOI: 10.1016/j.laa.2008.08.026.

Institute of Mathematics, Warsaw University, Banacha 2, 02-097 Warsaw, Poland

E-mail address: jmatczuk@mimuw.edu.pl

Department of Mathematical Sciences, Salman Farsi University of Kazerun, Kazerun, Iran, P.O. Box 73175-457.

E-mail address: ali.majidinya@gmail.com and ali.majidinya@kazerunsfu.ac.ir 